\title{
IDENTIFIED APLYSIA NEURONS FORM SPECIFIC CHEMICAL SYNAPSES IN CULTURE ${ }^{1}$
}

\author{
JOSEPH CAMARDO, ERIC PROSHANSKY, AND SAMUEL SCHACHER ${ }^{2}$ \\ Departments of Anatomy and Psychiatry, Center for Neurobiology and Behavior, College of Physicians \& Surgeons, Columbia \\ University, and The New York State Psychiatric Institute, New York, New York 10032
}

Received May 20, 1983; Accepted July 21, 1983

\begin{abstract}
Identified neurons from the abdominal ganglion of the marine mollusc Aplysia californica make specific transmitter-mediated synapses in dissociated cell culture. The cholinergic interneuron L10 makes synapses in vitro with one group of its follower cells, the left upper quadrant cells, and these connections exhibit the features of these synapses in vivo when the postsynaptic cells are plated with their initial axon segments. Furthermore, L10 will avoid making synapses with right upper quadrant cells, which contain cholinergic receptors but do not synapse with L10 in vivo.

This in vitro system can therefore be used as a model in which to study the development of specific neuronal connections.
\end{abstract}

Two of the fundamental features of nervous system function are the establishment of precise connections between neurons (Sperry, 1963; Jacobson, 1978) and the capacity for the strength of these connections to be modified (Tsukahara, 1981; Kandel and Schwartz, 1982). In many invertebrate species, the ability to map out the connections of single identified cells has contributed greatly to our understanding of synaptic physiology (Kandel, 1976; Hoyle, 1977; Krasne, 1978). Invertebrates have also been used to study neuronal development (Goodman and Pearson, 1982). In some species, the ability to identify and follow neurons through embryonic life has been an important tool in the exploration of cell lineage and growth (Sulston and Horvitz, 1977; Weisblat et al., 1978; Goodman and Spitzer, 1979). However, the development of synaptic specificity and plasticity has

\footnotetext{
${ }^{1}$ We wish to thank R. Woolley, K. Hilten, and Louise Katz for their technical assistance, T. Capo, S. Perritt, and J. Paige at the Marine Biologial Laboratory at Woods Hole for providing the animals used in this study, and Harriet Ayers for help with the manuscript. We also thank E. Kandel, C. Bailey, V. Castellucci, and R. Ambron for their comments on earlier drafts of the manuscript. The research was supported by National Institutes of Health Grant NS19216 and National Science Foundation Grant 79223602 to S. S., Sloan Foundation Postdoctoral Fellowship to E. P., National Institute of Mental Health Training Grant MH15174 to J. C., and National Institutes of Health Grant GM32099 which supports the mariculture facility at the Marine Biological Laboratory.

2 To whom correspondence should be addressed, at Center for Neurobiology and Behavior, College of Physicians and Surgeons, Columbia University, and New York State Psychiatric Institute-Annex, 722 West 168 Street, New York, 10032.
}

not been directly examined in these preparations. In other species, such as molluscs, in which intracellular recording is easily done in the adult, it is difficult to perform experiments on identified cells until rather late in development, long after specific connections have been established.

Recently, however, several investigators have reported that identified neurons of some invertebrate species, including Aplysia, Helisoma, and leech, can be maintained in culture, and that under these conditions neurons can re-establish functional electrical or chemical connections (Kaczmarek et al., 1979; Ready and Nicholls, 1979; Dagan and Levitan, 1981; Fuchs et al., 1981, 1982; Wong et al., 1981; Schacher et al., 1982). Cell culture has been extremely useful in the study of synapse development in vertebrate preparations, such as the neuromuscular junction and sympathetic ganglia (Furshpan et al., 1976; Fischbach and Nelson, 1977; Patterson, 1978). Synapse formation in culture between identified neurons of invertebrates would permit the study of specific central connections as they are being made.

Wc rcport here that, in cell culture, neuron L10 of Aplysia will make specific synapses with appropriate target neurons and will not make synapses with inappropriate neurons, even though the non-target neurons contain receptors to the neurotransmitter released by the presynaptic cell. L10 is a cholinergic, multiaction interneuron which exerts a dual inhibitory effect on cells L2 through L6 (left upper quadrant cells, LUQ cells): a fast IPSP, mediated by an increase in conductance to chloride, and a slow IPSP, elicited by repetitive stimulation, which is thought to result from either an increase in 
conductance to $\mathrm{K}^{+}$or a decrease in conductance to $\mathrm{Na}^{+}$ (Kunze and Brown, 1971; Kandel and Gardner, 1972; Kehoe, 1972; Wilson and Wachtel, 1979). In culture, L10 will make this dual inhibitory-inhibitory connection onto LUQ cells dissociated with an initial segment of axon. It elicits only the slow component of the postsynaptic potential (PSP) onto LUQ cells cultured as isolated somata.

\section{Materials and Methods}

Tissue culture procedures. The methods used for plating the cells have been described (Schacher and Proshansky, 1983). Abdominal ganglia from juvenile Aplysia ( 1 to $3 \mathrm{gm}$ ) raised from fertilization at the Marine Biological laboratory at Woods Hole, MA (Kriegstein et al., 1974; Kriegstein, 1977) were bathed for $2 \mathrm{hr}$ at $34^{\circ} \mathrm{C}$ in $1 \%$ protease (Sigma type IX) in L15 medium (Flow Laboratories, McLean, VA) plus appropriate salts. Following removal of the sheath, the cells were isolated from the ganglia with glass microelectrodes in one of two ways: either the cell body alone was isolated, or the cell was removed with an initial segment of axon (Schacher and Proshansky, 1983). L10, left upper quadrant cells L2 to L6 (LUQ cells), and right upper quadrant cells R3 to R13 (RUQ cells) were identified on the basis of size, position, and pigmentation (Koester and Kandel, 1977). Each LUQ and RUQ cell was treated as a member of a homogeneous population, since L10 in vivo connects with all cells in the LUQ cluster (dual component IPSP) and does not connect with any RUQ cell (Kandel et al., 1967; Koester and Kandel, 1977). Cells were transferred individually into poly-L-lysine-coated culture dishes containing 50\% L15 plus salts and 50\% sterile-filtered Aplysia hemolymph (Schacher and Proshansky, 1983). The culture dishes contained one of three different groups of cells; ( $a$ ) one L10 and two to three LUQ cells; $(b)$ one L10 and two to three RUQ cells; or $(c)$ one L10, two LUQ, and two RUQ cells. To ensure that cells remained close to each other and could be identified for subsequent electrophysiological examination, the culture dishes were allowed to remain on the dissecting microscope platform for $24 \mathrm{hr}$. The cultures were then transferred to a $20^{\circ} \mathrm{C}$ incubator. The medium was changed every 2 days with the same original stock used at initial plating.

Electrophysiology and iontophoresis. All intracellular recording was done with a single-barrelled electrode of 5 to 15 megohms filled with $2 \mathrm{M}$ potassium citrate or $2 \mathrm{M}$ $\mathrm{KCl}$. Acetylcholine $(\mathrm{ACh})$ was applied either by iontophoresis from a 50-megohm electrode filled with $1 \mathrm{M}$ $\mathrm{ACh}$, or from a microelectrode broken to 500 kilohms, filled with $50 \mu \mathrm{M} \mathrm{ACh}$, which was brought up to the cell body or processes to elicit a response by diffusion from the pipette tip.

\section{Results}

L10 forms two types of chemical connections with LUQ cells. By $48 \mathrm{hr}$ in culture, the cells regenerate an overlapping network of neurite processes (Fig. 1). Chemical synapses between L10 and LUQ cells are re-established in about $80 \%$ of the cultures after 3 to 5 days (Table I). In all cases the connection is inhibitory: action potentials in L10 hyperpolarize the LUQ cells. There are, however, differences in the electrophysiological properties of the connection that depend on the manner in which the LUQ cells are initially plated.

L10 forms the dual inhibitory-inhibitory connection characteristic of this synapse in vivo onto LUQ cells plated with their initial axons (Fig. 2, $A$ to $D$ ). The fast component of the PSP begins 20 msec after the upstroke of the action potential in L10 and lasts $800 \mathrm{msec}$, and the reversal potential is $77 \pm 2 \mathrm{mV}(n=4)$. The slow component is elicited after several spikes have fired and lasts for several seconds after the stimulus. The slow potential is nulled but not reversed by hyperpolarization. In addition, the reversal potential of the fast component is shifted in the depolarizing direction by intracellular injection of chloride ions $(n=4)$. The reversal potential of the slow IPSP is not affected by increasing the intracellular chloride concentration (Fig. 2D).

When LUQ cells are plated without axons, L10 forms a connection which lacks the fast component (Fig. $2 E$ ). A single spike in L10 elicits no response in the LUQ cell, but repetitive firing elicits a slow, long-lasting hyperpolarization. The IPSP is nulled but not reversed with hyperpolarization. This distinctive characteristic of the slow component was found in seven of eight cultures in which the LUQ cells had no axonal segments (see Table I). In one culture, a nonreversible slow IPSP was evoked by a single spike.

LUQ cells have acetylcholine receptors. The L10-LUQ synapse in vivo is cholinergic (Giller and Schwartz, 1971; Eisenstadt et al., 1973), and the formation of an L10-

\section{TABLE I}

Specific synapse formation between $L 10$ and LUQ cells

Summary of cultures examined electrophysiologically 3 to 5 days after plating for the presence of chemical synapses between the presynaptic neuron L10 and LUQ cells (target) or RUQ cells (non-target). In all cases L10 was presented with two to three IUQ or RUQ cells. Group I consists of cultures containing L10 with LUQ cells. The LUQ cells were plated either as isolated cell bodies or with axons. The reversible fast component that is normally seen at this connection in vivo is elicited when LUQ cells are plated with axons (see Fig. 2). Group II consists of cultures containing L10 with RUQ cells or L10 with both LUQ and RUQ cells plated either with or without axons. No significant L10-evoked response is detectable in the RUQ cells in either type of cultures (see the text and Figs. 3 and 4). Each culture dish is counted as having a synapse if at least one connection is present. The total number of synapses is counted separately since, in some cultures, L10 made synapses with more than one LUQ cell.

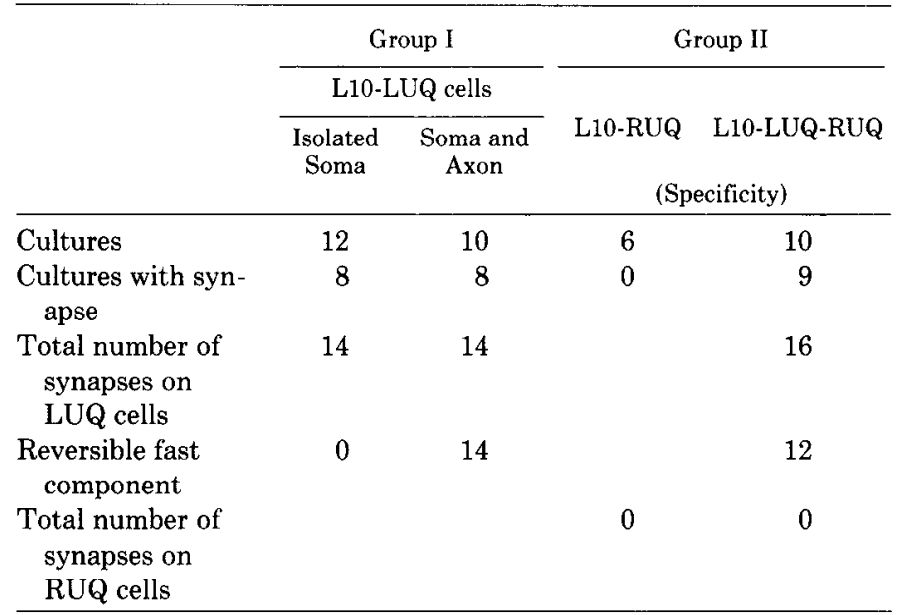



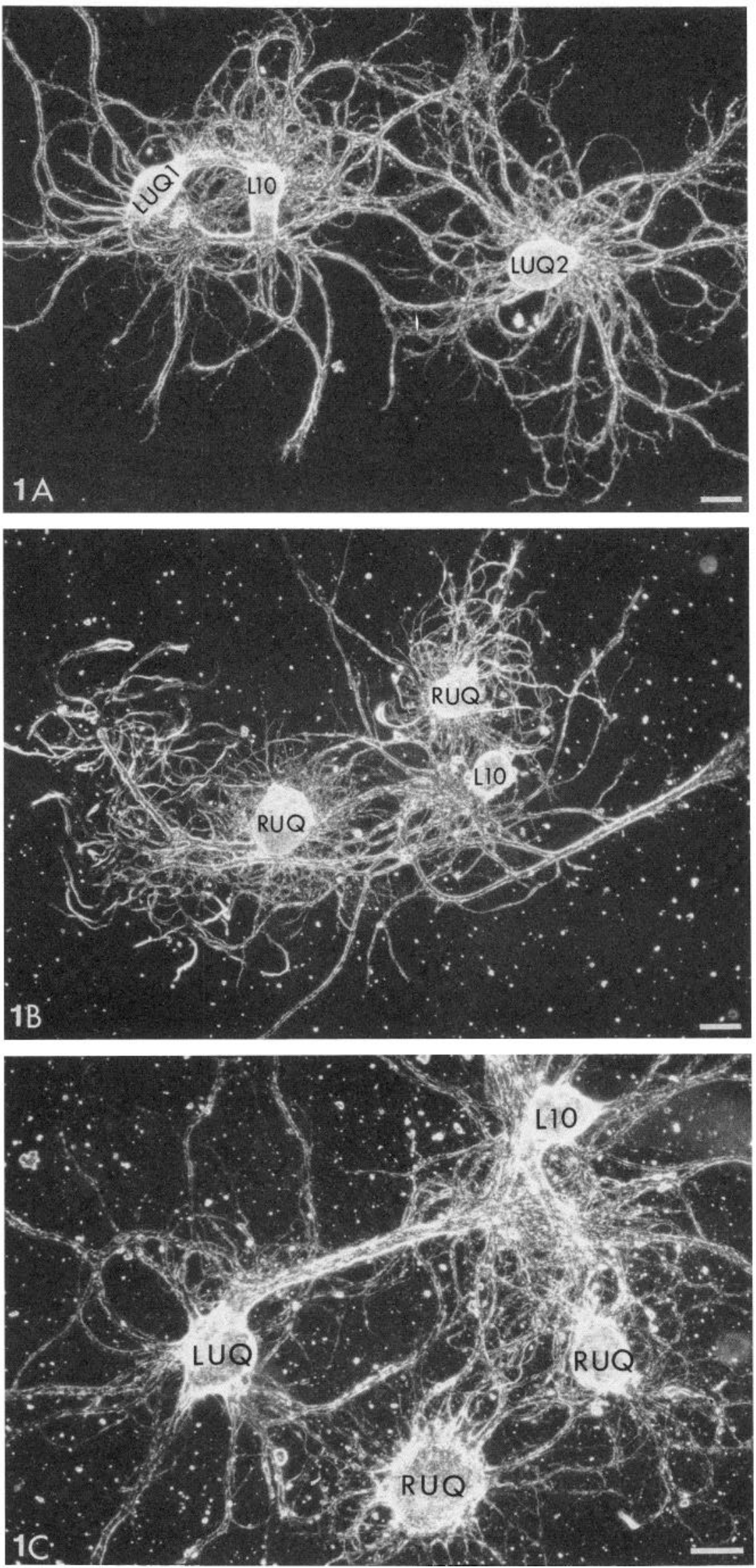

Figure 1 

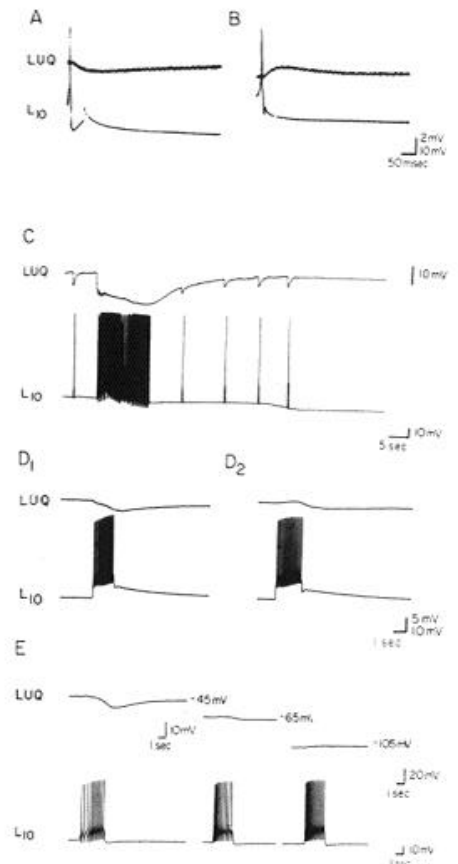

Figure 2. L10 forms chemical synapses with LUQ cells plated with axons $(A$ to $D)$ or without axons $(E)$. $A$ and $B$, A single spike in L10 elicits an IPSP onto the LUQ cell after 3 days in culture. The latency of the response is approximately $20 \mathrm{msec}$ and the duration is $800 \mathrm{msec}$. In $B$, this IPSP is reversed when the LUQ cell is hyperpolarized to $-50 \mathrm{mV}$ below the resting potential (resting potential is $-50 \mathrm{mV}$ ) and $-25 \mathrm{mV}$ below the reversal potential. $C, \mathrm{~L} 10$ forms a dual-component PSP on LUQ cells after 5 days in culture. A single spike elicits the fast PSP, and repetitive firing elicits both the fast PSP and, with a latency of 2 to $3 \mathrm{sec}$, a slow IPSP. The latter outlasts the stimulus by several seconds and recovers gradually. Single spikes in L10 again elicit the fast PSP during the recovery of the slow potential. $D$, The fast component of the PSP is sensitive to intracellular chloride ion concentration. In $D_{1}$, repetitive stimulation of $\mathrm{L} 10$ elicits a fast component accompanied by a slow component in the LUQ cell impaled initially with a potassium citrate electrode. In $D_{2}$, the same LUQ cell is impaled with a potassium chloride electrode, and hyperpolarizing current is passed for several minutes to inject chloride. The fast IPSP is reversed at the resting potential, but the slow IPSP is unaffected. $E$, L10 forms only the slow component PSP on a LUQ cell plated without its axon, after 5 days in culture. A single spike in L10 elicits no measurable response from the LUQ cell. With repetitive firing, a slow response can be seen at the resting potential (left trace). This response is nulled when the LUQ cell is hyperpolarized by $-20 \mathrm{mV}$ (center trace), but does not reverse with further hyperpolarization (right trace).
LUQ connection with properties identical to that seen in vivo suggests that the in vitro connection is also cholinergic. We tested this by examining the response of LUQ cells to ACh. LUQ cells which have formed the dual inhibitory synapse with L10 respond to ACh with a fast and slow IPSP; the fast IPSP is reversible. This response is evoked on the cell body or regenerated neurites with iontophoresis, and by diffusion from a pipette filled with a concentration of ACh as low as $50 \mu \mathrm{M}$. Since this is exactly the response evoked by L10, it is consistent with the idea that L10 in culture releases ACh.

We also examined synapses made by L10 onto LUQ cells without axons. In this case, despite the fact that L10 elicits only a slow PSP, ACh elicits both components of the ACh response. Furthermore, the dual inhibitory response is evoked by ACh onto LUQ cells which are cultured in isolation. These observations suggest that LUQ cells maintain normal receptors regardless of the presence of a synaptic connection.

L10 forms specific chemical connections. Our data indicate that L10 will re-establish normal connections in vitro when presented with one set of its usual target neurons. Our next experiments were designed to ask whether L10 will retain the ability to recognize and make synapses exclusively with its usual target cells. We therefore prepared two different kinds of cultures. First, L10 was cultured with RUQ cells, with which it does not synapse in vivo. These cells are easily identifiable and, like LUQ cells, respond in vivo to ACh (Koester and Kandel, 1977). We find that RUQ cells (cell body and regenerated processes) will also respond in vitro to $\mathrm{ACh}$ (see Fig. 3, $E$ and $F$ ) and the amplitude of the RUQ cell response to ACh is comparable to that seen in LUQ cells. Second, L10 was cultured with LUQ cells and RUQ cells simultaneously to see if the presynaptic neuron could select its usual target from a heterogeneous population of cells, all of which make receptors to the transmitter released by L10.

No significant interaction is seen between L10 and RUQ cells even though the regenerated processes of the RUQ cells clearly overlap the processes of L10 (Fig. 1, $B$ and $C$ ). In most cultures (14 of 16), stimulation of L10 evoked no response in the RUQ cells (Table I, Figs. 3 and 4). In 2 of 16 cultures, high frequency firing of L10 (10 to $15 \mathrm{~Hz}$ ) could elicit a slow hyperpolarization from one of the RUQ cells of less than $1 \mathrm{mV}$. Even in these cultures, however, the weak response in the RUQ cells is labile; L10 stimulation often produced no response in the RUQ cells.

Figure 1. Darkfield photomicrographs of L10 co-cultured with LUQ cells, RUQ cells, or both LUQ and RUQ cells after 4 days in culture. The bars equal $100 \mu \mathrm{m}$. A, L10 with two LUQ cells. The LUQ cell on the left (1) was plated with an axon whereas the LUQ cell on the right (2) was plated initially without an axon. Electrophysiological examination of the synapses formed showed the dual component PSP on LUQ 1 and the single slow component PSP on LUQ 2. B, L10 with two RUQ cells. All cells were plated initially without axons. No chemical connection formed. Note that the processes of L10 overlap with those of the RUQ cells. C, L10 with LUQ and RUQ cells. All cells were plated initially with axons (including a second LUQ cell not seen in this field). L10 made synapses only with the LUQ cells even though RUQ cell processes overlap with L10 processes. 
A

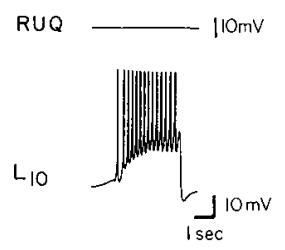

B

LUQ w $10 \mathrm{mV}$

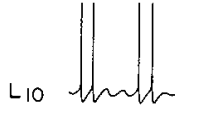

$\underset{i \sec }{\operatorname{din} \mathrm{mV}}$

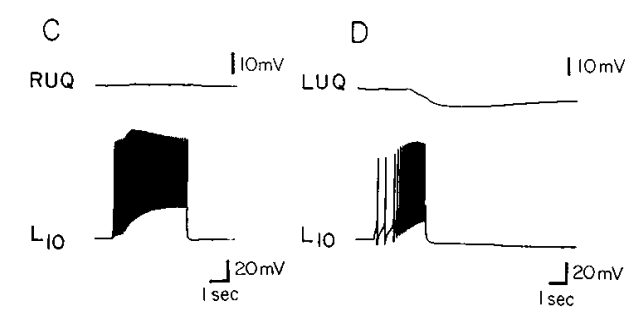

E

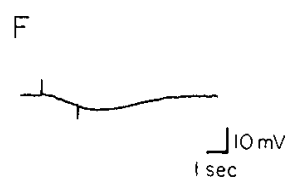

Figure 3. L10 forms synapses with LUQ cells and not with RUQ cells. $A$ and $B, \mathrm{~L} 10, \mathrm{RUQ}$, and LUQ cells are plated with initial axons. After 4 days, spikes in L10 elicit no response in the RUQ cell. The same L10 elicits an IPSP from the LUQ cell. $C$ and $D$, In this culture, the LUQ and RUQ cells are plated without axons. After 4 days, repetitive firing of the L10 elicits no response from the RUQ cells, but a slow IPSP is elicited in the LUQ cell by an equal stimulus to the same L10. $E$ and $F$, Response by a RUQ cell to iontophoresis of ACh on cell body $(E)$ and regenerated processes $(F)$. The hyperpolarizing response to $\mathrm{ACh}$ is the same as the response to $\mathrm{ACh}$ in vivo. The iontophoretic pipette in $F$ is placed in an area of the neurite network where, under phase contrast microscopy, the RUQ neurites could be seen to overlap those of L10.

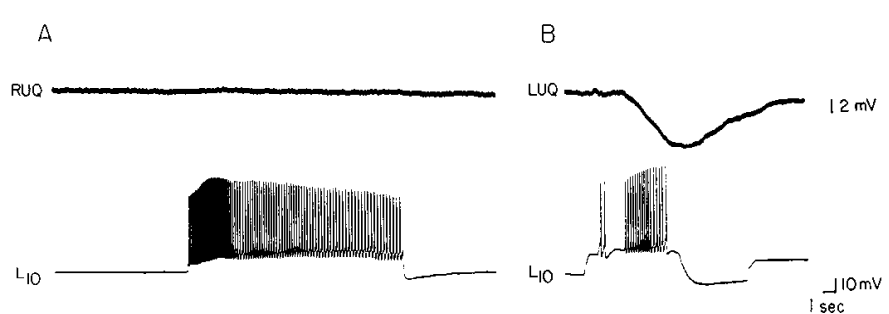

Figure 4. Comparison of response of RUQ cell and LUQ cell to repetitive stimulation of $\mathrm{L} 10$, at high gain. $A, \mathrm{~L} 10$ is fired for scveral scconds at a high rate and elicits no observable change in potential of the RUQ cell. $B$, The response of the LUQ cell to repetitive firing of the same L10 is dramatic and immediate. This trace shows both summated fast IPSPS and the long-lasting IPSP.

When L10 is cultured with both target cells (LUQ cells) and non-target cells (RUQ cells), as shown in Figure $1 C, \mathrm{~L} 10$ makes synapses with the LUQ cells and avoids the RUQ cells. The synapse is specific whether or not the LUQ and RUQ cells have initial axon segments, and no significant interaction is observed between L10 and RUQ cells even though strong connections are made between L10 and LUQ cells in the same dish (Figs. 3 and 4). The results of these experiments indicate that the L10-LUQ synapse is a specific physiologic interaction.
L10 will make no effective connection when presented with the wrong target, and will select the appropriate target neuron when presented with both correct and incorrect target cells.

\section{Discussion}

We conclude from these experiments that isolated, identified neurons in Aplysia will re-establish specific connections in culture. The neuron L10 and the target cells L2 to L6 from juvenile animals retain the ability to make a specific chemical connection, despite the availability of other non-target cells which make receptors to the transmitter released by L10. We emphasize that our results demonstrate electrophysiologically functional synaptic interactions; the morphology and pharmacology of the actual contact have not yet been examined. Furthermore, the connection between L10 and LUQ cells is normal if the LUQ cells are cultured with a segment of axon. Without the initial axon, L10-LUQ connections show only the slow PSP. The key finding of this study is the demonstration of non-random, physiologically significant interactions between specific pairs of cells with properties comparable to the synapses in the intact nervous system.

Although the formation of the L10-LUQ connection in vitro is clear, a number of questions regarding the mechanisms remain unanswered. We cannot account for the observation that the formation of the dual IPSP requires an axon on the LUQ cell. It is obviously not the result of the absence of the receptor-ionophore responsible for the chloride IPSP, since the fast response can be evoked by ACh regardless of the nature of the synapse. However, it is possible that LUQ cells, without their axons, will not cluster the receptors at release sites. Our methods of $\mathrm{ACh}$ iontophoresis could not detect this. Alternatively, the fast PSP may require a postsynaptic specialization that requires an initial axonal segment to develop. Schacher and Proshansky (1983) have observed that neurite regeneration and morphology can be profoundly altered by the presence or absence of an initial axon segment, and we may be observing a physiologic effect of this alteration.

A second important aspect of these experiments is that the L10-LUQ connection is a non-random interaction. We suggest that $\mathrm{L} 10$ in vitro is able to recognize the difference between its usual target neurons and nontarget neurons when the two groups are presented either together or separately, and that L10 will synapse specifically with its target cells. The implication is that this process is similar to that which occurs during the formation of specific neural connections in vivo. However, there are other possible explanations for the lack of an observable L10-RUQ connection in culture, not directly related to specificity per se. For example, if an L10-RUQ connection were to form which was electrically remote from the cell body, we would not detect it using our electrophysiological methods. Neither could we detect a synapse if the receptors on RUQ cells were, for some reason, not clustered appropriately at the L10 release sites. Although this would still result in a specific connection, the mechanism may not involve cell-cell recognition. We favor the idea that the synapse is formed 
specifically, but we cannot completely rule out these other explanations without morphological data which show the absence of any specialized L10-RUQ contacts.

One other question is raised by our data. We find that, in some cases ( 2 of 16), with repetitive stimulation, L10 will evoke a weak and labile hyperpolarization onto RUQ cells. Although this is neither an effective nor a physiologically significant interaction, compared to the L10LUQ connection formed in culture, there are different ways to account for it. First, L10 terminals could release transmitter before making any synaptic contact and, if a RUQ neurite is nearby, ACh will diffuse to it and evoke a response. If this is true, it is remarkable that we see this result so rarely, and it suggests that some process is at work even in culture to actively prevent this type of nonspecific interaction. There is, however, an alternative. L10 could make synapses transiently with every cell in culture, and the mechanism of specificity may be the elimination of inappropriate synapses, as has been observed in other developing systems (Changeaux and Danchin, 1976; Ruffolo et al., 1978; Purves and Lichtman, 1980). Since we make only one observation in time (between 3 and 5 days), we may, in most cases, see only those connections which remain after the synapses have been sorted out. The weak L10-RUQ interactions may therefore be contacts which have not yet been eliminated. We cannot distinguish these possibilities on the basis of our data, but the question immediately suggests experiments to examine the time course of synapse development in vitro.

On the basis of the data presented here, we suggest that the formation of the L10-LUQ connection in vitro parallels the development of this synapse in vivo. It is therefore an appropriate model in which to study factors which contribute to synaptic specificity. The advantage of this system is that it consists of a small number of large, uniquely identifiable neurons which can be studied electrophysiologically and biochemically (Ambron et al., 1982) during the actual formation of the synapse. Furthermore, since this synapse in vivo shows both posttetanic potentiation and presynaptic inhibition, it is possible that the development of these features of the synapse can also be examined in vitro.

\section{References}

Ambron, R., S. Schacher, A. Silverman, and E. Proshansky (1982) Identified neurons in culture: Protein synthesis and distribution during neurite outgrowth. J. Cell Biol. 95: 48a.

Changeaux, J. P., and A. C. Danchin (1976) Selective stabilization of developing synapses as a mechanism for the specification of neuronal networks. Nature 264: 705-712.

Dagan, D., and I. B. Levitan (1981) Isolated identified Aplysia neurons in cell culture. J. Neurosci. 1: 736-740.

Eisenstadt, M., J. E. Goldman, E. R. Kandel, H. Hoike, J. Koester, and J. H. Schwartz (1973) Intrasomatic injection of radioactive precursors for studying transmitter synthesis in identified neurons of Aplysia californica. Proc. Natl. Acad. Sci. U. S. A. 70: 3371-3375.

Fischbach, G. D., and P. G. Nelson (1977) Cell culture in neurobiology. In Handbook of Physiology, Vol. 1: The Nervous System, J. M. Brookhart and V. B. Mountcastle, eds., pp. 719 774, American Physiological Society, Bethesda, MD.
Fuchs, P. A., J. G. Nicholls, and D. F. Ready (1981) Membrane properties and selective connections of identified leech neurons in culture. J. Physiol. (Lond.) 316: 203-224.

Fuchs, P. A., L. P. Henderson, and J. G. Nicholls (1982) Chemical transmission between individual Retzius and sensory neurons of the leech in culture. J. Physiol. (Lond.) 323: 195-210.

Furshpan, E. J., P. R. MacLeish, P. H. O'Lague, and D. D. Potter (1976) Chemical transmission between rat sympathetic neurons and cardiac myocytes developing in microcultures. Proc. Natl. Acad. Sci. U. S. A. 73: 4225-4229.

Giller, E., and J. II. Schwartz (1971) Choline acetyltransferase in the identified neurons of abdominal ganglion of Aplysia californica. J. Neurophysiol. 34: 93-107.

Goodman, C. S., and K. F. Pearson (1982) Neuronal development: Cellular approaches in invertebrates. Neurosci. Res. Program Bull. 20: 773-942.

Goodman, C. S., and N. C. Spitzer (1979) Embryonic development of identified neurons. Nature 280: 208-214.

Hoyle, G. (1977) Identified Neurons and Behavior of Arthropods, Plenum Press, New York.

Jacobson, M. (1978) Developmental Neurobiology, Plenum Press, New York.

Kaczmarek, L., M. Finbow, J. P. Revel, and F. Strumwasser (1979) The morphology and coupling of Aplysia bag cells within the abdominal ganglion and in cell culture. J. Neurobiol. 10: $535-550$.

Kandel, E. R. (1976) Cellular Basis of Behavior, W. H. Freeman \& Co., San Francisco.

Kandel, E. K., and D. Gardner (1972) 'Ihe synaptic actions mediated by the different branches of a single neuron. In Neurotransmitters: Proceedings, Association for Research in Nervous and Mental Diseases, E. J. Kopin, ed., Vol. 50, pp. 91-144, The Williams \& Wilkins Co., Baltimore.

Kandel, E. R., and J. H. Schwartz (1982) Molecular biology of learning: Modulation of transmitter release. Science 218: $433-443$.

Kandel, E. R., W. T. Frazier, R. Waziri, and R. E. Coggeshall (1967) Direct and common connections among identified neurons in Aplysia. J. Neurophysiol. 30: 1352-1376.

Kehoe, J. S. (1972) Ionic mechanisms of a two component cholinergic inhibition in Aplysia neurons. J. Physiol. (Lond.) 225: 85-114.

Koester, J., and E. R. Kandel (1977) Further identification of neurons in the abdominal ganglion of Aplysia using behavioral criteria. Brain Res. 121: 1-20.

Krasne, F. B. (1978) Extrinsic control of intrinsic neuronal plasticity: An hypothesis from work on simple systems. Brain Res. 140: 197-215.

Kriegstein, A. R. (1977) Developmeni of the nervous system of Aplysia californica. Proc. Natl. Acad. Sci. U. S. A. 74: 375378.

Kriegstein, A. R., V. Castellucci, and E. R. Kandel (1974) Metamorphosis of Aplysia californica in laboratory culture. Proc. Natl. Acad. Sci. U. S. A. 71: 3654-3658.

Kunze, D. L., and A. M. Brown (1971) Internal potassium and chloride activities and the effects of acetylcholine on identifiable Aplysia neurons. Nature New Biol. 229: 229-231.

Patterson, P. H. (1978) Environmental determination of autonomic neurotransmitter functions. Annu. Rev. Neurosci. 1: 1-17.

Purves, D., and J. W. Lichtman (1980) Elimination of synapses in the developing nervous system. Science 210: 153-157.

Ready, D. F., and J. G. Nicholls (1979) Identified neurons isolated from leech CNS make selective connections in culture. Nature 182: 67-69.

Ruffolo, R. R., S. S. Eisenbarth, J. M. Thompson, and M. Nirenberg (1978) Synapse turnover: A mechanism for ac- 
quiring specificity. Proc. Natl. Acad. Sci. U. S. A. 75: 22812285.

Schacher, S., and E. Proshansky (1983) Neurite regeneration by Aplysia neurons in dissociated cell culture: Modulation by Aplysia hemolymph and the presence of the initial axonal segment. J. Neurosci. 3: 2403-2413.

Schacher, S., E. Proshansky, and J. S. Camardo (1982) Neurite outgrowth and synapse formation by identified Aplysia neurons in dissociated cell culture. Soc. Neurosci. Abstr. 8: 17.

Sulston, J. E., and H. R. Horvitz (1977) Post-embryonic cell lineages of the nematode Caenorhabditis elegans. Dev. Biol. 56: 100-156.

Sperry, R. W. (1963) Chemoaffinity in the orderly growth of nerve fiber patterns and connections. Proc. Natl. Acad. Sci.
U. S. A. 50: 703-710.

Tsukahara, N. (1981) Synaptic plasticity in the mammalian central nervous system. Annu. Rev. Neurosci. 4: 351-380.

Weisblat, D. A., R. T. Sawyer, and G. S. Stent (1978) Cell lineage analysis by intracellular injection of tracer enzyme. Science 202: 1295-1298.

Wilson, W. P., and H. Wachtel (1979) Prolonged inhibition in burst firing neurons: Synaptic inactivation of the slow regenerative inward current. Science 202: 772-775.

Wong, R. G., R. D. Hadley, S. B. Kater, and G. C. Hauser (1981) Neurite outgrowth in molluscan organ and cell cultures: The role of conditioning factor(s). J. Neurosci. 1 : 10081021. 